impossible to suspect their presence. All the others gave symptoms, but in no case was a correct diagnosis made. The rupture may be taken for ectopic gestation, renal colic, perforative appendicitis, or other forms of peritonitis. Pain in the right side with fulness of the flank and possibly signs of internal hemorrhage may here give one the correct clue. The tumor caused by the false aneurysm is also very difficult to diagnose. The condition with which it is most likely to be confused is a renal new growth. It may also be mistaken for hydronephrosis and cystic kidney. A new growth will have a more progressive character, especially in regard to general ill health. Enlarged veins, varicocele or metastasis may clear up the diagnosis. Hydronephrosis is usually more elastic, if not fluctuating, and varies in size simultaneously with the passage of large quantities of urine. At certain stages it is very painful. Pulsation cannot be depended on as a diagnostic feature, but a bruit can be heard in most cases and should be looked for.

Prognosis.-The prognosis without operation is most unfavorable. Some of the patients with smaller true aneurysms died from other causes, but all patients having false aneurysms died from the diseased condition, except one in whom the aneurysm was successfully removed by an operation. Two other patients were operated on and died. One of these cases should not be classed as operative, since the operation has been performed because of injuries, and death was due to these, while the aneurysm had been present for some time previously without giving symptoms.

In my case, death was due to the inability of the opposite kidney to provide for the body, and not as a direct result of the operation. The causes of death were profuse hemorrhage from rupture into the renal pelvis, hemorrhage into the peritoneal cavity, syncope, and exhaustion. In several cases, the causes were from rupture and extravasation of blood in the flank. In some cases, the cause of death was not stated. The length of time during which symptoms were present varied from a few days to five years. If the primary rupture does not kill the patient, it may go on increasing slowly for many months or years until the tumor becomes of enormous size.

Treatment.-The renal artery presents one place in the body where the part supplied by the artery can be wholly removed without producing serious consequences. The only prospect of saving life is by nephrectomy and the extirpation of the aneurysmal sac. In the recent cases and those with a small tumor this can be best done by the flank; but in cases of a large tumor it can best be managed by the transperitoneal route. The difficulties of the operation are considerable, as the adhesions may be dense and the tissues extensively infiltrated with blood clot. The operation will probably be undertaken for some other than the correct condition, and the correct diagnosis may not be at once recognized. An attempt should be made to tie off the artery before disturbing much of the blood clot, or alarming hemorrhage may be encountered. If the patient's condition can bear it, the sac and kidney should be removed. The dangers are hemorrhage and shock. In all cases in which operation was performed, the shock was considerable and required energetic treatment. There is also danger of injury to some of the abdominal contents when the mass is large and the adhesions firm.

\section{THE DISTRIBUTION OF MENINGOCOCCI IN THE UPPER RESPIRATORY TRACT OF CARRIERS *}

\author{
R. D. HERROLD, M.D. \\ CHICAGO
}

This study was undertaken to compare the frequency of positive cultures from the nasopharynx in meningococcus carriers with their frequency from other parts of the upper respiratory tract, and to determine the relation, if any, that such other parts may have to the persistence of the cocci in the nasopharynx. No reference could be found in the literature to any observations on these points.

During an extensive experience with the detection of meningococcus carriers in a large body of men under control, ninety-three segregated men, without discrimination in selection, were examined soon after isolation with regard to the presence of meningococci elsewhere than in the nasopharynx.

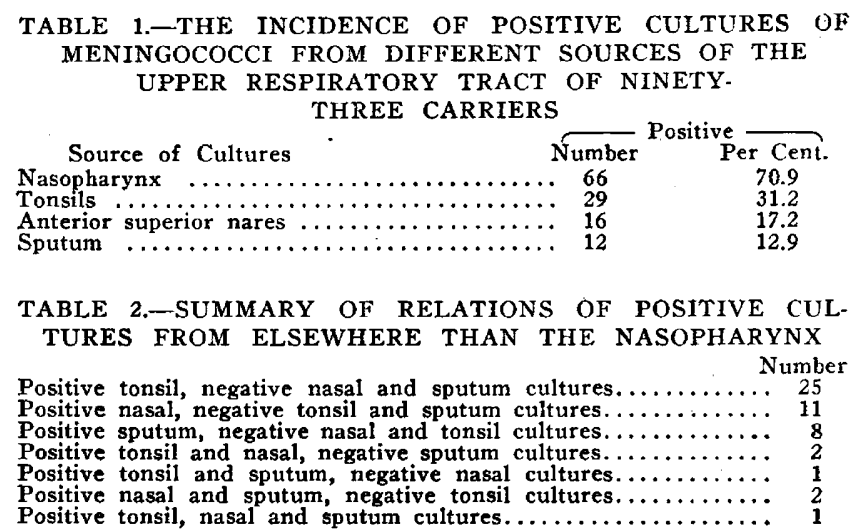

TABLE 3.-A COMPARISON OF THE PERSISTENCE OF THE CARRIER STATE ACCORDING TO THE DISTRIBUTION OF MENINGOCOCCI

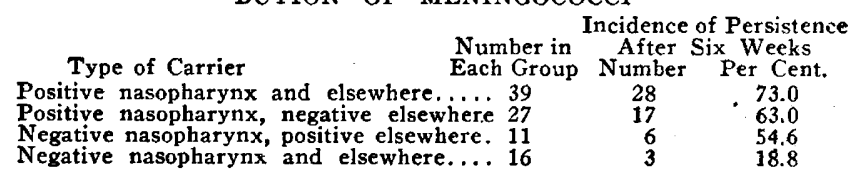

Cultures were made of the nasopharynx, the tonsils, the anterior superior nares, and the sputum of each individual on the same day. The examinations extended over several weeks as the carriers were detected. A sterile wire swab, with the tip bent at an angle of 30 degrees, was introduced through the mouth; the end was passed over the posterior wall of the nasopharynx and removed with care not to touch any other surface. The medium used was plain blood agar made as follows: Approximately 15 to 20 drops of defibrinated goat's blood were added to 10 c.c. of ordinary nutrient agar for each Petri dish. The tip of the swab was rubbed on a small area near the edge of the blood agar plate, and then streaks were made from this inoculation. With ordinary straight swabs, smears were made from the tonsils and anterior superior nares, and inoculations made as described. In case of the sputum cultures there is always the danger of contamination from the mouth. After forced coughing and expectoration into a sterile Petri dish, a loopful of material, which appeared to have come from the

\footnotetext{
* From the Memorial Institute for Infectious Diseases.
} 
deeper bronchi, was fished out and streaked on the blood agar plate. Only those sputum cultures are reported as positive which give many colonies of meningococci, these being in most instances the predominating organisms. All cultures diagnosed as positive were agglutinated in a dilution of $1: 100$ or higher of a polyvalent antimeningococcus serum, control tests with normal horse serum giving no agglutination. Fermentation tests with strains from these carriers gave results typical of meningococci.

In Table 1 , the relative incidence of positive cultures are summarized. The apparently large number of negative cultures from the nasopharynx is what would be expected for two reasons: First, a large percentage of newly isolated carriers will give negative cultures within a few days, and secondly, many chronic carriers give intermittent negative cultures. An analysis of Table 3 brings out the fact that a greater number of carriers persist as such after six weeks in those groups in which other parts than the nasopharynx give positive cultures.

\section{CONCLUSIONS}

The nasopharynx is the most common seat of meningococci in carriers, but the carrier state persists longer, the wider the distribution of meningococci in the upper respiratory tract. The number of positive cultures from other sources than the nasopharynx seems sufficient to warrant the taking of cultures from other parts than only the nasopharynx.

\section{Clinical Notes, Saggestions, and New Instruments}

\section{CARE OF THE EYES OF THE PATIENT DURING ETHERIZATION \\ Albert H. Miller, M.D., Providence, R. I.}

It is fortunate that conjunctivitis is an infrequent postanesthetic complication as, when it occurs, the pain resulting from the conjunctivitis is likely to be so severe as to outweigh any other discomfort that may follow the operation. Conjunctivitis is supposed to follow exposure of the eyes to ether vapor, or to result from the spilling of the liquid anesthetic on them. In the anesthetic literature, mention of this question is limited to directions for covering the eyes with a towel, gauze handkerchief, or a strip of rubber tissue for the purpose of protection from the anesthetic vapor. In cases in which these precautions have been carefully taken, conjunctivitis occasionally develops. In a series of 3,000 cases of ether anesthesia by the open method in which the eyes were not covered, although they were exposed to the ether vapor and although liquid ether was without doubt accidentally spilled over the eyes in some cases, no conjunctivitis has occurred.

If the anesthetist allows a drop of liquid ether to be placed in his own eye, no severe reaction follows. Ethyl chlorid is equally harmless to the eye. If a quantity of liquid ether is accidentally spilled over the uncovered but closed eyes of the anesthetized patient, conjunctivitis does not usually follow. When the towel or gauze covering the eyes becomes saturated with ether and remains in this condition, a severe conjunctivitis will probably result. In case the patient's eyes do not completely close during anesthesia, or are held open by a carelessly applied protective covering, conjunctivitis is very likely to occur. There can be no doubt that the precautions usually taken to avoid postanesthetic conjunctivitis in reality increase the danger and often are the sole cause of the trouble.

The only conclusion to be drawn is that, during general anesthesia, the eyes should not be covered. If liquid ether is accidentally spilled over the eyes, they may be washed with saline solution or sterile water, or the lids may be carefully dried with soft gauze. The lids should be closed during anesthesia. In the cases in which there is tendency for them to remain open, they should be frequently closed with the tip of the finger. The lid reflex may be frequently tested with the finger tip and at the same time the condition of the pupil noted. The unclean and unnecessary practice of touching the conjunctiva to elicit the corneal reflex should rarely if ever be indulged in. By this simple technic, the eyes may be safeguarded during general anesthesia.

131 Waterman Street.

DISLOCATION OF THE MIDDLE CUNEIFORM BONE

Frank Warner, M.D., Columbus, Ohio

Fellow of the American College of Surgeons

Dislocation of the middle cuneiform bone occurs so rarely that instances of its occurrence should be reported. Its rarity adds to the difficulty of recognizing the condition; but

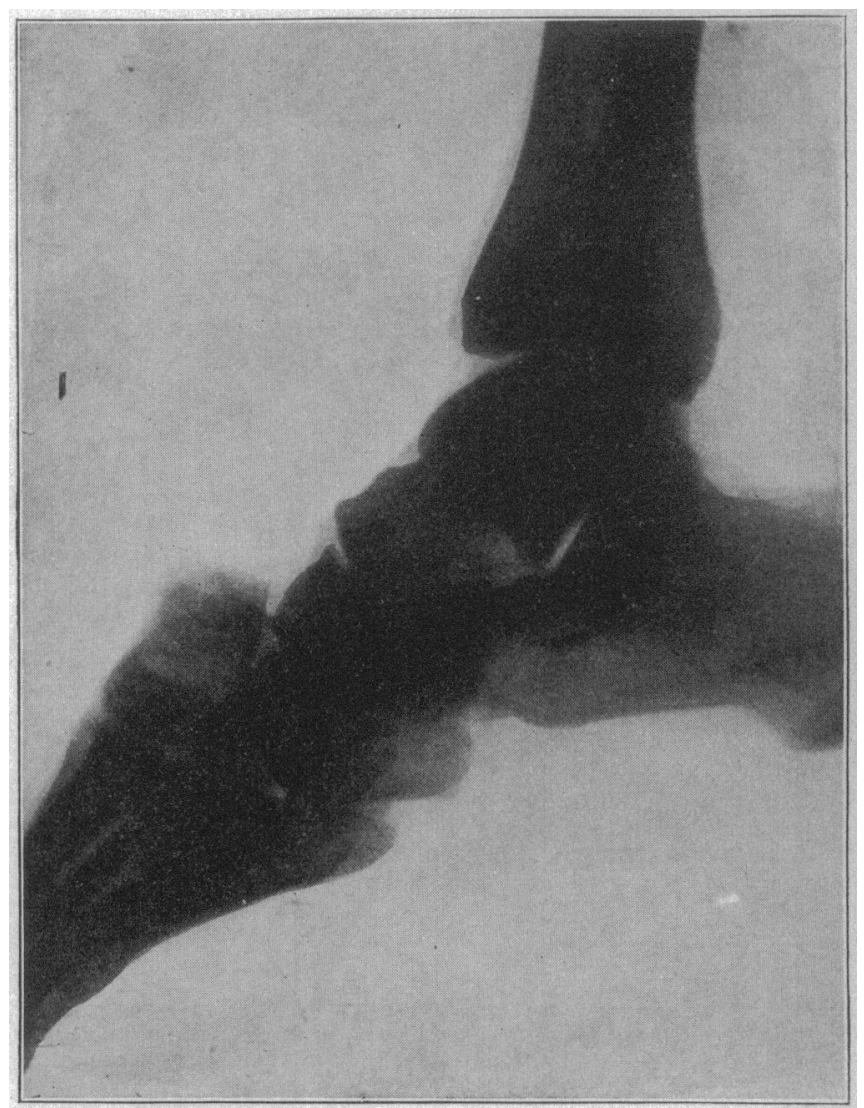

Fig. 1.-Dislocation of the middle cuneiform bone of the left foot.

unless it iso recognized, impaired usefulness of the foot will result. Stimson ${ }^{1}$ was able to collect only seven instances of separate dislocations of the middle cuneiform bone, and eleven of fractures. All three cuneiform bones have been displaced together, and the second and third likewise.

March 2, 1917, V. G. E., aged 28, a railway conductor, while wearing a pair of heavy work shoes with extra thick, double soles, had his left foot run over by an empty dump car. The skin was not lacerated, but there was a bony protuberance on the dorsal surface, over the situation of the middle cuneiform. A diagnosis of dislocation of this bone was confirmed by roentgenoscopy. Failing to reduce the dislocation, first without an anesthetic and then with one, I made an incision over the protuberance, and skidded the bone back into position on a pry, when the torn ligaments were sutured together. The

1. Stimson, L. A.: A Practical Treatise on Fractures and Disloca. tions, Ed. 7, Philadelphia, Lea and Febiger, 1912, p. 914. 